\title{
Preoperative Controlling Nutritional Status plus Tumor Burden Score for the Assessment of Prognosis after Curative Liver Resection for Hepatocellular Carcinoma
}

\author{
Yasuyuki Fukami Takuya Saito Takaaki Osawa Takashi Arikawa \\ Tatsuki Matsumura Shintaro Kurahashi Shunichiro Komatsu Kenitiro Kaneko \\ Tsuyoshi Sano
}

Division of Gastroenterological Surgery, Department of Surgery, Aichi Medical University, Nagakute, Japan

\section{Highlights of the Study}

- The prognostic predictive value of adding the tumor factor (i.e., Tumor Burden (TB) score) to the Controlling Nutritional Status (CONUT) score for patients with hepatocellular carcinoma (HCC) has not been reported previously.

- This study showed that the preoperative CONUT plus TB (CONUT-TB) score is a valuable predictor of survival in patients with HCC undergoing liver resection.

\section{Keywords}

Hepatocellular carcinoma · Controlling Nutritional Status score $\cdot$ Tumor burden score $\cdot$ Liver resection · Prognostic factor

\begin{abstract}
Objective: There are no previous studies analyzing the prognostic predictive value of adding the tumor factor (i.e., Tumor Burden (TB) score) to the Controlling Nutritional Status (CONUT) score for patients with hepatocellular carcinoma (HCC). This study aimed to investigate the value of the CONUT plus TB (CONUT-TB) score as a prognostic predictor in patients with HCC undergoing liver resection. Methods: Between 2015 and 2018, 96 consecutive patients with HCC underwent liver resection at our institution. Patients undergoing repeated liver resection and combined resection of a metastatic lesion were excluded. Patients were divided into
\end{abstract}

2 groups according to their CONUT-TB scores according to a cutoff value. Clinicopathologic prognostic factors for survival were analyzed using a database containing the medical records. Results: The optimal cutoff value of the CONUT-TB score determined by using a minimum $p$ value approach was 13 points. Among the 81 patients included in the analytic cohort, 71 patients had low $(<13)$ and 10 patients had high (>13) CONUT-TB scores. The overall 3-year survival rate of patients following liver resection for $\mathrm{HCC}$ in the high-CONUT-TB group was significantly worse than that of patients in the low-CONUT-TB group ( 62.5 vs. $89.3 \%, p=0.003$ ). Multivariate analysis indicated that a high CONUT-TB score was independently associated with overall survival after liver resection $(p=0.010)$. Conclusion: The CONUT-TB score is a valuable predictor of survival in patients with HCC after liver resection.
(C) 2020 The Author(s)

Published by S. Karger AG, Basel karger@karger.com www.karger.com/mpp

Karger $\stackrel{\text { ' }}{5}$

GOPEN ACCESS
(C) 2020 The Author(s)

Published by S. Karger AG, Basel

This is an Open Access article licensed under the Creative Commons Attribution-NonCommercial-4.0 International License (CC BY-NC) (http://www.karger.com/Services/OpenAccessLicense), applicable to the online version of the article only. Usage and distribution for commercial purposes requires written permission.
Yasuyuki Fukami

Division of Gastroenterological Surgery, Department of Surgery

Aichi Medical University

1-1 Yazakokarimata, Nagakute, Aichi 480-1195 (Japan)

yasuyuki490225@yahoo.co.jp 


\section{Introduction}

Hepatocellular carcinoma (HCC) is one of the most common malignancies and is estimated to be responsible for nearly 745,000 deaths per year $[1,2]$. Liver resection has been a mainstay of treatment for HCC in patients with well-preserved liver function and early-stage HCC [3]. Advances in diagnostic imaging and widespread application of screening programs in high-risk populations have improved the detection of HCC at early stages.

Numerous clinical and pathological factors have been proposed for the estimation of the prognosis of patients with HCC after liver resection. In particular, tumor size and the number of lesions have similarly been utilized to predict long-term outcomes for patients with $\operatorname{HCC}[4,5]$. Mazzaferro et al. [5, 6] demonstrated that the tumor size and number criteria for HCC have undergone numerous iterations culminating in a predictive tool termed the "metro-ticket" system. Furthermore, Sasaki et al. [7, 8] showed that the "metro-ticket" concept was applicable to patients undergoing liver resection for colorectal liver metastases, and they established a novel scoring system (i.e., Tumor Burden (TB) score).

On the other hand, the controlling nutritional status (CONUT) score [9] is a useful tool to evaluate not only the immune-nutritional status but also long-term outcomes after liver resection of HCC [10-12]. Although tumor-related factors and preoperative immune-nutritional status are important factors affecting long-term survival in patients with HCC, no previous study has assessed a scoring system combining them. This study aimed to investigate the effect of the CONUT plus TB (CONUT$\mathrm{TB}$ ) score as a novel prognostic predictor in patients with HCC after liver resection.

\section{Methods}

In total, 96 consecutive patients with HCC underwent liver resection at our institution between January 2015 and December 2018. Liver resection was defined as the complete removal of tumors detected by preoperative imaging or intraoperative inspection using ultrasonography. After review by a multidisciplinary board, all HCC tumors were assessed for resectability by hepatobiliary surgeons. $\mathrm{Pa}$ tients undergoing repeated liver resection $(n=14)$ and combined resection of a metastatic lesion $(n=1)$ were excluded. Clinicopathologic prognostic factors for survival were analyzed using a database containing medical records. Major liver resection was defined as the resection of 3 or more Couinaud's segments. All patients provided signed informed consent before undergoing therapy. This study was approved by the Institutional Review Board of our institution (No. 2019-007) based on the Helsinki Declaration of 1964 and its later amendments or comparable ethical standards.

\section{Perioperative Management}

For tumor staging, abdominal ultrasonography and enhanced computed tomography or magnetic resonance imaging were performed before treatment. Hepatic angiography and positron emission tomography scans were not routinely used. Treatment types were determined by the treatment algorithm for HCC proposed by the Japanese guidelines [13]. All patients were evaluated for the possibility of liver resection. The indications for liver resection were based on an algorithm that included the presence/absence of ascites, serum total bilirubin levels, and results of indocyanine green clearance tests $[13,14]$.

During laparotomy, adequate exploration and intraoperative ultrasonography of the liver were routinely performed to detect previously unsuspected liver lesions and to assess the possibility of resection. Parenchymal transection was performed using either Péan forceps or a Cavitron Ultrasonic Surgical Aspirator (CUSA) under intermittent total hepatic inflow vascular clamping for 20 min at 5-min intervals. Any complications that developed within 90 days after the operation were recorded.

Tumor staging was performed according to the 8th edition of the American Joint Committee on Cancer (AJCC)/International Union against Cancer (UICC) Staging Manual [15] and the Barcelona Clinic Liver Cancer (BCLC) Staging System $[3,16]$.

\section{Preoperative CONUT Score}

Preoperative CONUT scores were calculated based on previous reports according to 3 parameters which were the serum albumin concentrations, peripheral lymphocyte counts, and total cholesterol concentrations (Table 1) [9].

\section{TB Score}

The TB score was calculated by combining the tumor size and the total number of tumors from the final pathologic specimen. Specifically, the TB score was defined as the distance from the origin on a Cartesian plane that incorporated 2 variables: maximum tumor size ( $x$-axis) and the number of liver lesions ( $y$-axis). The Pythagorean theorem was then used to calculate the distance of any given point from the origin of the plane $(0,0)$, whereby $\left[\mathrm{TBS}^{2}=\right.$

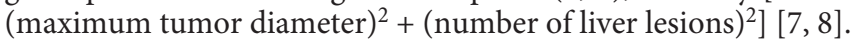

\section{CONUT-TB Score}

The CONUT-TB score was defined as the sum of the CONUT and TB scores.

\section{Postoperative Follow-Up}

The patients underwent ultrasonography and measurements of tumor markers every 3 or 4 months and enhanced computed tomography or magnetic resonance imaging at least every 6 or 12 months, according to the protocol stated by the Japanese guidelines [12]. For patients who experienced tumor recurrence after liver resection, repeated liver resection was considered for those without any medical contraindications for liver surgery, including liver functional reserve and future remnant liver volume. If repeated liver resection could not be performed due to poor liver condition or other unfavorable factors, then transarterial chemoembolization, radiofrequency ablation, or systemic chemotherapy was employed.

During the follow-up period, 21 patients (26\%) developed radiologically detectable tumor recurrence. All patients received additional treatment as follows: transarterial chemoembolization 
Table 1. Scoring system according to CONUT

\begin{tabular}{lcccc}
\hline Parameter & \multicolumn{2}{l}{ Malnutrition status } & \\
\cline { 2 - 5 } & normal & light & moderate & severe \\
\hline Serum albumin, g/dL & $\geq 3.5$ & $3.0-3.49$ & $2.5-2.99$ & $<2.5$ \\
Score & 0 & 2 & 4 & 6 \\
Lymphocyte count, /mm & $\geq 1,600$ & $1,200-1,599$ & $800-1,199$ & $<800$ \\
Score & 0 & 1 & 2 & 3 \\
Total cholesterol, mg/dL & $\geq 180$ & $140-179$ & $100-139$ & $<100$ \\
Score & 0 & 1 & 2 & 3 \\
CONUT score $^{\mathrm{a}}$ & $0-1$ & $2-4$ & $5-8$ & $9-12$ \\
\hline
\end{tabular}

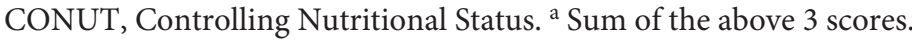

Table 2. Patient characteristics

\begin{tabular}{lc}
\hline & $N=81$ \\
\hline Age, years & $70(29-89)$ \\
Sex (male/female) & $65 / 16$ \\
BMI, kg/m² & $22.8(13.6-31.3)$ \\
Hepatitis B virus infection & $17(21 \%)$ \\
Hepatitis C virus infection & $26(32 \%)$ \\
ASA status (I/II/III) & $39 / 42 / 0$ \\
AFP, ng/mL & $10.3(1.3-86,586)$ \\
PIVKA-II, mAU/mL & $62(10-429,000)$ \\
ICGR15, \% & $11.1(2.4-35.4)$ \\
Child-Pugh score (A/B) & $79 / 2$ \\
CONUT score & $5(2-21)$ \\
Major liver resection (>3 segments) & $15(19 \%)$ \\
Laparoscopic resection & $23(28 \%)$ \\
Operative duration, minduration, min & $276(119-787)$ \\
Total blood loss, mL & $281(1-7,822)$ \\
Surgical margin (R0/R1) & $77 / 4$ \\
Tumor size, cm & $3(1-15)$ \\
Tumor number (solitary/multiple) & $69 / 12$ \\
Poor differentiation & $10(12 \%)$ \\
Microvascular invasion & $19(23 \%)$ \\
Histological liver cirrhosis & $27(33 \%)$ \\
UICC stage (IA/IB/II/IIIA/IIIB) & $14 / 35 / 24 / 7 / 1$ \\
BCLC stage (0/A/B) & $14 / 55 / 12$ \\
\hline
\end{tabular}

Expressed as $n$ (\%) or median (range). BMI, body mass index; ASA, American Society of Anesthesiologists; AFP, alpha-fetoprotein; PIVKA-II, Prothrombin induced by vitamin K absence-II; ICGR15, indocyanine green retention rate at $15 \mathrm{~min}$; CONUT, Controlling Nutritional Status; UICC, Union for International Cancer Control; BCLC, Barcelona Clinic Liver Cancer.

( $n=12)$, repeat liver resection $(n=4)$, radiation $(n=2)$, lymph node resection $(n=1)$, radiofrequency ablation $(n=1)$, and sorafenib $(n=1)$ administration.

Data Acquisition

By accessing patient records, we were able to obtain the following clinical data: patient age, sex, $\mathrm{BMI}$, hepatitis $\mathrm{B} / \mathrm{C}$ virus infec- tion, American Society of Anesthesiologists (ASA) status, alphafetoprotein, prothrombin induced by vitamin $\mathrm{K}$ absence-II, indocyanine green retention rate at $15 \mathrm{~min}$, Child-Pugh score, surgical procedure, operative duration, total blood loss, tumor size, tumor number, histopathologic features of the resected tumor, morbidity, follow-up time, and survival time.

\section{Statistical Analysis}

The continuous data are expressed as medians (ranges). The categorical variables are expressed as number (percentage). The statistical analyses were performed using $\chi^{2}$ tests, Mann-Whitney U tests, or Fisher's exact probability tests, as appropriate. The overall survival of patients was determined from the time of surgery to the time of death or the most recent follow-up. Overall survival curves were generated by the Kaplan-Meier method, and differences in survival rates were compared using the log-rank test. The variables identified as potentially significant by univariate analysis were subsequently chosen for multivariate analysis with the Cox proportional hazards model to identify the independent predictors of survival. The assumptions of proportional hazards were checked by the time-dependent Cox regression model. The variables identified as potentially significant by univariate analysis ( $p$ value $<0.20$ ) were selected for multivariate analysis with a logistic regression model to identify the independent predictors of postoperative major complications. All $p$ values were 2 -sided, and $p<0.05$ was considered to indicate a statistically significant difference. Concerning the impact of CONUT-TB scores on survival, the "minimum $p$ value" approach, which was performed using the log-rank test for the overall survival, was used to determine the best cutoff values of each score for predicting survival for the entire cohort. All statistical calculations were performed using the IBM SPSS Statistics 21 software package (IBM Japan Inc., Tokyo, Japan).

\section{Results}

Clinical characteristics of the entire cohort are shown in Table 2. At the time of operative exploration, the median age of all patients was 70 years, and the patient cohort included 65 men and 16 women. The median CONUT score was 5 points (range: $2-21$ points). Major liver resection and laparoscopic resection were performed 


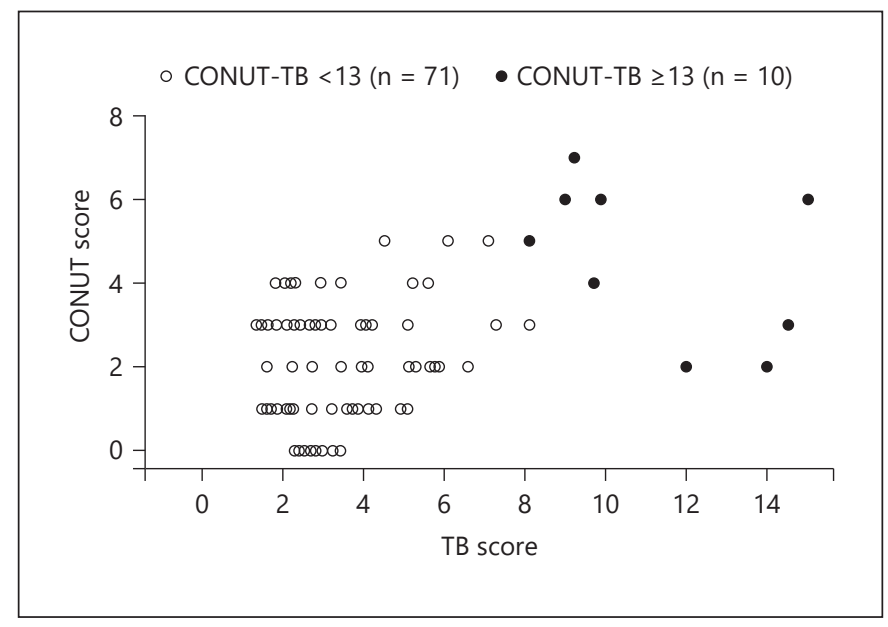

Fig. 1. Distributions of the CONUT score and TB score. CONUT, Controlling Nutritional Status; TB, Tumor Burden.

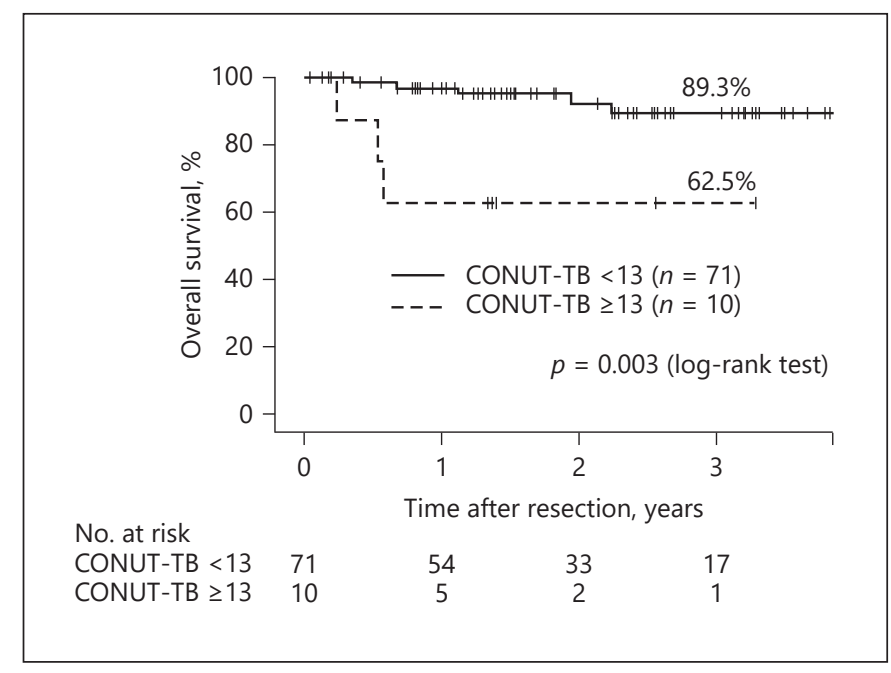

Fig. 3. Overall survival in 81 patients who underwent liver resection for HCC stratified according to low or high CONUT-TB scores. CONUT-TB, Controlling Nutritional Status plus Tumor Burden; HCC, hepatocellular carcinoma.

in 15 and 23 patients (19 and 28\%), respectively. The median tumor size was 3 (range: $1-15) \mathrm{cm}$. Twelve patients had multiple HCC. The median follow-up period after diagnosis was 18 (range: 1-48) months. The distributions of the CONUT scores and TB scores are shown in Figure 1.

The best cutoff value of CONUT-TB for predicting survival was determined by using a minimum $p$ value

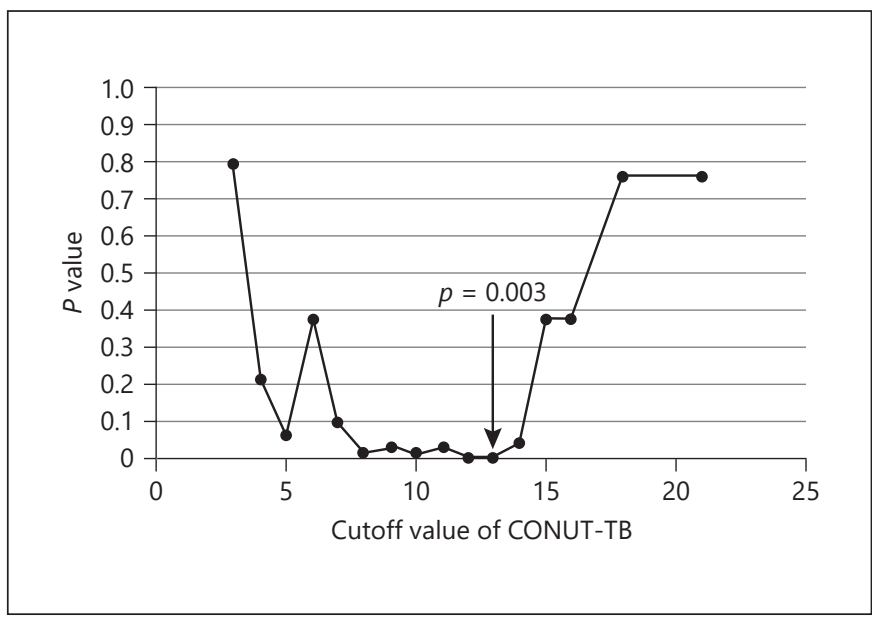

Fig. 2. Optimal cutoff value of CONUT-TB for predicting overall survival. When testing CONUT-TB cutoff values from 2 to 21 , the minimum $p$ value was obtained at 13 (arrow). CONUT-TB, Controlling Nutritional Status plus Tumor Burden.

approach for the entire cohort. When sliding the cutoff value of CONUT-TB from 2 to 21 , the minimum $p$ values were obtained at 13 (Fig. 2). Table 3 shows the comparison of the clinicopathologic features of CONUT-TB $<13(n=71)$ and $\geq 13(n=10)$. The prothrombin induced by vitamin $\mathrm{K}$ absence-II levels was significantly higher in the high CONUT-TB group than in the low CONUT-TB group. There were significant differences between the 2 groups in the major liver resection rates ( $11 \%$ in the low vs. $70 \%$ in the high) and tumor sizes (3 $\mathrm{cm}$ in the low vs. $10 \mathrm{~cm}$ in the high). The in-hospital and 90-day postoperative mortality rates were zero in both groups.

The overall 3-year survival rate of patients following liver resection for HCC in the high CONUT-TB group was significantly worse than that of patients in the low CONUT-TB group (62.5 vs. $89.3 \%, p=0.003$ ) (Fig. 3 ). Multivariate analysis (Table 4) indicated that a high CONUT-TB score (hazard ratio: 6.72, 95\% CI: 1.59-28.40, $p=0.010)$ was independently associated with overall survival after liver resection.

\section{Discussion}

This study identified an association between longterm survival and a high CONUT-TB score (i.e., 13 points) in patients who underwent liver resection for HCC. To the best of our knowledge, this is the first report on the prognostic value of the combination of the CO- 
Table 3. Clinicopathological features of patients according to the CONUT-TB

\begin{tabular}{|c|c|c|c|}
\hline & $\begin{array}{l}\text { CONUT-TB }<13 \\
(n=71)\end{array}$ & $\begin{array}{l}\text { CONUT-TB } \geq 13 \\
(n=10)\end{array}$ & $p$ \\
\hline Age, years & $70(29-89)$ & $73(52-85)$ & 0.672 \\
\hline Sex (male/female) & $55 / 16$ & $10 / 0$ & 0.198 \\
\hline BMI, $\mathrm{kg} / \mathrm{m}^{2}$ & $22.8(13.6-31.3)$ & $23.3(17.4-26.0)$ & 0.943 \\
\hline $\mathrm{AFP}, \mathrm{ng} / \mathrm{mL}$ & $9.2(1.3-86,586)$ & $21.4(2.1-3,314)$ & 0.145 \\
\hline PIVKA-II, mAU/mL & $46(10-151,000)$ & $27,490(27-429,000)$ & $<0.001$ \\
\hline ICGR15, \% & $11.1(2.4-35.4)$ & $13.9(6.2-17.7)$ & 0.514 \\
\hline Child-Pugh score (A/B) & $70 / 1$ & $9 / 1$ & 0.233 \\
\hline Major liver resection ( $\geq 3$ segments) & $8(11 \%)$ & $7(70 \%)$ & $<0.001$ \\
\hline Laparoscopic resection & $23(32 \%)$ & 0 & 0.055 \\
\hline Operative duration, $\min$ & $268(119-635)$ & $333(194-787)$ & 0.084 \\
\hline Total blood loss, mL & $261(1-7,822)$ & $333(203-2,856)$ & 0.084 \\
\hline Surgical margin (R0/R1) & $68 / 3$ & $9 / 1$ & 0.416 \\
\hline \multicolumn{4}{|l|}{ Morbidity } \\
\hline Wound infection & $3(4 \%)$ & $1(10 \%)$ & 0.416 \\
\hline Intraabdominal abscess & $2(3 \%)$ & $1(10 \%)$ & 0.330 \\
\hline Bile leakage & $3(4 \%)$ & $1(10 \%)$ & 0.416 \\
\hline Pleural effusion & $7(10 \%)$ & $1(10 \%)$ & 1.000 \\
\hline Refractory ascites & $1(1 \%)$ & $2(20 \%)$ & 0.039 \\
\hline Liver failure & 0 & 0 & - \\
\hline Other & $11(15 \%)$ & $2(40 \%)$ & 0.659 \\
\hline Mortality & 0 & 0 & - \\
\hline Tumor size, $\mathrm{cm}$ & $3(1-8)$ & $10(5-15)$ & $<0.001$ \\
\hline Tumor number (solitary/multiple) & $62 / 9$ & $7 / 3$ & 0.163 \\
\hline Poor differentiation & $7(10 \%)$ & $3(30 \%)$ & 0.103 \\
\hline Microvascular invasion & $13(18 \%)$ & $6(60 \%)$ & 0.009 \\
\hline Histological liver cirrhosis & $26(37 \%)$ & $1(10 \%)$ & 0.153 \\
\hline UICC stage (IA/IB/II/IIIA/IIIB) & $14 / 30 / 21 / 5 / 1$ & $0 / 5 / 3 / 2 / 0$ & 0.414 \\
\hline BCLC stage $(0 / \mathrm{A} / \mathrm{B})$ & $14 / 48 / 9$ & $0 / 7 / 3$ & 0.153 \\
\hline
\end{tabular}

Expressed as $n(\%)$ or median (range). CONUT-TB, Controlling Nutritional Status plus Tumor Burden; BMI, body mass index; AFP, alpha-fetoprotein; PIVKA-II, Prothrombin induced by vitamin K absence-II; ICGR15, indocyanine green retention rate at $15 \mathrm{~min}$; UICC, Union for International Cancer Control; BCLC, Barcelona Clinic Liver Cancer.

Table 4. Univariate and multivariate analyses for overall survival after liver resection

\begin{tabular}{|c|c|c|c|c|c|c|}
\hline \multirow[t]{2}{*}{ Variable } & \multicolumn{3}{|c|}{ Univariate } & \multicolumn{3}{|c|}{ Multivariate } \\
\hline & HR & $95 \% \mathrm{CI}$ & $p$ & HR & $95 \% \mathrm{CI}$ & $p$ \\
\hline Age $70 \geq$ years & 0.77 & $0.16-3.59$ & 0.737 & & & \\
\hline Sex (female) & 1.06 & $0.11-10.59$ & 0.962 & & & \\
\hline PIVKA-II $\geq 400 \mathrm{mAU} / \mathrm{mL}$ & 1.03 & $0.14-7.39$ & 0.975 & & & \\
\hline Operative duration $\geq 300 \mathrm{~min}$ & 0.54 & $0.09-3.13$ & 0.491 & & & \\
\hline Total blood loss $\geq 500 \mathrm{~mL}$ & 1.61 & $0.32-8.02$ & 0.560 & & & \\
\hline Tumor differentiation (poor) & 0.66 & $0.07-6.56$ & 0.719 & & & \\
\hline Histological liver cirrhosis & 0.47 & $0.05-4.46$ & 0.507 & & & \\
\hline Curability R1 (vs. R0) & 5.77 & $0.51-64.90$ & 0.156 & & & \\
\hline CONUT-TB $\geq 13$ & 8.69 & $1.01-74.75$ & 0.049 & 6.72 & $1.59-28.40$ & 0.010 \\
\hline
\end{tabular}

PIVKA-II, Prothrombin induced by vitamin K absence-II; CONUT-TB, Controlling Nutritional Status plus Tumor Burden. 
NUT score and TB score in patients who underwent liver resection for HCC.

The CONUT score can be calculated from 3 parameters which are easy to measure before surgery such as the serum albumin levels, lymphocyte counts, and serum cholesterol levels. The CONUT score was originally designed as a screening tool to easily and objectively assess a patient's nutritional status [9]. The serum albumin concentration is the main indicator of nutritional condition and is also associated with systemic inflammatory response. The systemic and chronic inflammatory response to viruses or tumors is also associated with a decreased serum albumin concentration and poor prognosis in patients with HCC [17]. The lymphocyte count is an important indicator of a patient's immunological status and plays a fundamental role in cancer immune surveillance [18]. A lower lymphocyte count is associated with poor prognosis in patients with $\mathrm{HCC}$ as it is indicative of an inadequate immune response to cancer [19]. The serum cholesterol level has also been reported to correlate with the progression of various cancers, including HCC $[20,21]$. Therefore, the CONUT score could reflect not only the immune-nutritional status but also the long-term outcome after liver resection for HCC [10-12].

Many oncological factors have been proposed to predict the prognosis of patients with HCC after liver resection. Several authors have previously proposed tumor morphologic factors, including tumor size and number, as important predictors of long-term outcomes for patients with HCC. In fact, various cutoff values for the maximum tumor diameter and number have been analyzed to predict long-term outcomes for patients with HCC $[4,5,22]$. The "metro-ticket" system method of predicting survival was established to evaluate the feasibility of liver transplantation for patients with HCC [5, 6]. Recently, Sasaki et al. [7, 8] found that the "metroticket" concept was applicable to patients undergoing liver resection for colorectal liver metastases, and they established a novel scoring system (i.e., TB score). The TB score is a useful and accurate tool that can account for the impact that tumor morphology has on long-term outcomes in patients with HCC and colorectal liver metastases.

Although tumor-related factors and the preoperative immune-nutritional status are important factors affecting long-term survival in patients with HCC, no previous study has assessed the feasibility of combining them in a single scoring system. We hypothesized that the presence of a high CONUT-TB score is a predictive factor for poor long-term outcomes in patients who underwent liver resection for HCC. This study was designed to compare the long-term outcomes after liver resection in patients with a high CONUT-TB score versus a low CONUT-TB score of HCC. The overall survival rate was significantly worse in patients with a high CONUT-TB score than in those with a low CONUT-TB score. Regarding colorectal liver metastases, perioperative chemotherapy has expanded the indications for liver resection in addition to advanced multistep surgical techniques (i.e., portal vein embolization, 2-stage hepatectomy, and ALPPS) [23, 24]. The treatment principle for HCC is immediate surgery; therefore, the tumor factors cannot be changed. However, it may be possible to improve the immune-nutritional status before surgery.

Various methods of evaluating the immune-nutritional status have been developed, and recently, the Prognostic Nutritional Index (PNI) based on albumin levels and lymphocyte counts has been reported as a useful prognostic predictor for HCC $[25,26]$. However, the total cholesterol concentration is not included in the PNI. Therefore, the CONUT score is thought to be able to detect patients who will have a poor prognosis more sensitively than the PNI. In fact, Takagi et al. [10] demonstrated that the CONUT score was a more accurate prognostic factor than the PNI in patients undergoing liver resection for HCC. On the other hand, Pravisani et al. [27] reported that the CONUT score does not predict survival after liver transplantation in patients with HCC.

A limitation of this study is that it is a retrospectively designed study with a short follow-up time, and a propensity score matching analysis was not possible because the number of cases was too small in this single-center study. In the future, multicenter large series studies evaluating the oncological impact of the CONUT-TB score after liver resection for HCC would compensate for the limitations of this study.

\section{Conclusion}

Our study indicates that the CONUT-TB score is a convenient and valuable predictor of survival in patients with HCC after liver resection. Routine assessment of the CONUT-TB score could help manage meticulous followup after surgery. 


\section{Statement of Ethics}

This study was approved by the Institutional Review Board of our institution (No. 2019-007) and with the 1964 Helsinki declaration and its later amendments or comparable ethical standards.

\section{Conflict of Interest Statement}

The authors have no conflicts of interest to disclose.

\section{References}

1 Befeler AS, Di Bisceglie AM. Hepatocellular carcinoma: diagnosis and treatment. Gastroenterology. 2002;122(6):1609-19.

2 Ferlay J, Soerjomataram I, Dikshit R, Eser S, Mathers C, Rebelo M, et al. Cancer incidence and mortality worldwide: sources, methods and major patterns in GLOBOCAN 2012. Int J Cancer. 2015;136(5):E359-86.

3 Bruix J, Reig M, Sherman M. Evidence-based diagnosis, staging, and treatment of patients with hepatocellular carcinoma. Gastroenterology. 2016;150(4):835-53.

4 Zhong JH, Ke Y, Gong WF, Xiang BD, Ma L, Ye XP, et al. Hepatic resection associated with good survival for selected patients with intermediate and advanced-stage hepatocellular carcinoma. Ann Surg. 2014;260(2):329-40.

5 Mazzaferro V, Llovet JM, Miceli R, Bhoori S, Schiavo M, Mariani L, et al. Predicting survival after liver transplantation in patients with hepatocellular carcinoma beyond the Milan criteria: a retrospective, exploratory analysis. Lancet Oncol. 2009;10(1):35-43.

6 Mazzaferro V. Results of liver transplantation: with or without Milan criteria? Liver Transpl. 2007;13(11 Suppl 2):S44-7.

7 Sasaki K, Morioka D, Conci S, Margonis GA, Sawada Y, Ruzzenente A, et al. The tumor burden score: a new "metro-ticket" prognostic tool for colorectal liver metastases based on tumor size and number of tumors. Ann Surg. 2018;267:132-41.

8 Sasaki K, Margonis GA, Andreatos N, Zhang XF, Buettner S, Wang J, et al. The prognostic utility of the "Tumor Burden Score" based on preoperative radiographic features of colorectal liver metastases. J Surg Oncol. 2017;116(4): $515-23$.

9 Ignacio de Ulíbarri J, González-Madroño A, de Villar NG, González P, González B, Mancha A, et al. CONUT: a tool for controlling nutritional status. First validation in a hospital population. Nutr Hosp. 2005;20(1):38-45.

10 Takagi K, Yagi T, Umeda Y, Shinoura S, Yoshida R, Nobuoka D, et al. Preoperative controlling nutritional status (CONUT) score for assessment of prognosis following hepatectomy for hepatocellular carcinoma. World J Surg. 2017;41(9):2353-60.

11 Harimoto N, Yoshizumi T, Sakata K, Nagatsu A, Motomura T, Itoh S, et al. Prognostic significance of preoperative controlling nutritional status (CONUT) score in patients undergoing hepatic resection for hepatocellular carcinoma. World J Surg. 2017;41(11):280512.

12 Harimoto N, Yoshizumi T, Inokuchi S, Itoh S, Adachi E, Ikeda Y, et al. Prognostic significance of preoperative controlling nutritional status (CONUT) score in patients undergoing hepatic resection for hepatocellular carcinoma: a multi-institutional study. Ann Surg Oncol. 2018;25(11):3316-23.

13 Makuuchi M, Kokudo N, Arii S, Futagawa S, Kaneko S, Kawasaki S, et al. Development of evidence-based clinical guidelines for the diagnosis and treatment of hepatocellular carcinoma in Japan. Hepatol Res. 2008;38(1):3751.

14 Makuuchi M, Kosuge T, Takayama T, Yamazaki S, Kakazu T, Miyagawa S, et al. Surgery for small liver cancers. Semin Surg Oncol. 1993;9(4):298-304.

15 Amin MB, Edge S, Greene F, Byrd DR, Brookland RK, Washington MK, et al. AJCC cancer staging manual. 8th ed. New York: Springer; 2017.

16 Llovet JM, Brú C, Bruix J. Prognosis of hepatocellular carcinoma: the BCLC staging classification. Semin Liver Dis. 1999;19(3):32938.

17 Okamura Y, Ashida R, Ito T, Sugiura T, Mori K, Uesaka K. Preoperative neutrophil to lymphocyte ratio and prognostic nutritional index predict overall survival after hepatectomy for hepatocellular carcinoma. World J Surg. 2015;39(6):1501-9.

18 Coussens LM, Werb Z. Inflammation and cancer. Nature. 2002;420(6917):860-7.

19 Hu B, Yang XR, Xu Y, Sun YF, Sun C, Guo W, et al. Systemic immune-inflammation index predicts prognosis of patients after curative resection for hepatocellular carcinoma. Clin Cancer Res. 2014;20(23):6212-22.

20 Lee YL, Li WC, Tsai TH, Chiang HY, Ting CT. Body mass index and cholesterol level predict surgical outcome in patients with hepatocellular carcinoma in Taiwan: a cohort study. Oncotarget. 2016;7(16):22948-59.

21 Jiang SS, Weng DS, Jiang L, Zhang YJ, Pan K, Pan QZ, et al. The clinical significance of preoperative serum cholesterol and high-density lipoprotein-cholesterol levels in hepatocellular carcinoma. J Cancer. 2016;7(6):626-32.

22 Fukami Y, Kaneoka Y, Maeda A, Kumada T, Tanaka J, Akita T, et al. Liver resection for multiple hepatocellular carcinomas: a Japanese nationwide survey. Ann Surg. 2020; 272(1):145-54

23 Nordlinger B, Sorbye H, Glimelius B, Poston GJ, Schlag PM, Rougier P, et al. Perioperative chemotherapy with FOLFOX4 and surgery versus surgery alone for resectable liver metastases from colorectal cancer (EORTC Intergroup trial 40983): a randomised controlled trial. Lancet. 2008;371(9617):1007-16.

24 Fukami Y, Kurumiya Y, Kobayashi S. Associating liver partition and portal vein ligation for staged hepatectomy (ALPPS): an analysis of tumor activity. Updates Surg. 2014;66(3):223-5.

25 Man Z, Pang Q, Zhou L, Wang Y, Hu X, Yang $\mathrm{S}$, et al. Prognostic significance of preoperative prognostic nutritional index in hepatocellular carcinoma: a meta-analysis. HPB. 2018;20(10):888-95.

26 Chan AW, Chan SL, Wong GL, Wong VW, Chong CC, Lai PB, et al. Prognostic nutritional index (PNI) predicts tumor recurrence of very early/early stage hepatocellular carcinoma after surgical resection. Ann Surg Oncol. 2015;22(13):4138-48.

27 Pravisani R, Mocchegiani F, Isola M, Lorenzin D, Adani GL, Cherchi V, et al. Controlling Nutritional Status score does not predict patients' overall survival or hepatocellular carcinoma recurrence after deceased donor liver transplantation. Clin Transplant. 2020;34(3) e13786 